\title{
PROBABILITY FOR PRIMORDIAL BLACK HOLES IN HIGHER DERIVATIVE THEORIES
}

\author{
B. C. Paul* \\ Department of Physics, North Bengal University, \\ Siliguri, Dist. Darjeeling, Pin : 734 430, INDIA \\ Arindam Saha \\ Lataguri H. S. School, P.O. : Lataguri \\ Dist. : Jalpaiguri, West Bengal, Pin 735 219, INDIA
}

\begin{abstract}
The probability for quantum creation of an inflationary universe with a pair of black holes in higher derivative theories has been studied. Considering a gravitational action which includes quadratic $\left(\alpha R^{2}\right)$ and/or cubic term $\left(\beta R^{3}\right)$ in scalar curvature in addition to a cosmological constant $(\Lambda)$ in semiclassical approximation with Hartle-Hawking boundary condition, the probability has been evaluated. The action of the instanton responsible for creating such a universe, with spatial section with $S^{1} X S^{2}$ topology, is found to be less than that with a spatial $S^{3}$ topology, unless $\alpha<-\frac{1}{8 \Lambda}$ in $R^{2}$-theory. In the $R^{3}$ theory, however, there exists a set of solutions without a cosmological constant when $\beta R^{2}=1$ and $\alpha=-3 \sqrt{\beta}$ which admit primordial black holes $(\mathrm{PBH})$ pair in an inflationary universe scenario. We note further that when $\beta R^{2} \neq 1$, one gets PBH pairs in the two cases : (i) with $\alpha$ and $\Lambda$ both positive and (ii) with $\Lambda$ positive and $\alpha$ negative satisfying a constraint $6|\alpha| \Lambda>1$. However, the
\end{abstract}

*e-mail : bcpaul@nbu.ernet.in 
relative probability for creation of an inflationary universe with a pair of black holes in the $R^{3}$-theory suppresses when $\alpha>-2 \sqrt{\beta}$ or $|\alpha|<2 \sqrt{\beta}$. However, if the above constraints are relaxed one derives interesting results leading to a universe with $\mathrm{PBH}$ in $R^{3}$-theory without cosmological constant.

PACS No(s). : 04.20.Jb, 04.60.+n, 98.80.Hw 


\section{INTRODUCTION :}

In recent times black holes are the most elusive object to study in Astrophysics and Cosmology. The mass of these objects may be greater than the solar mass or even less. It is known in stellar physics that a blackhole is the ultimate corps of a collapsing star when its mass exceeds twice the mass of the sun. Another kind of black holes are becoming important in cosmology which might have formed due to quantum fluctuations of matter distribution in the early universe. These are topological blackholes having mass very small. In particular these are important from the view point of Hawking radiation [1]. Bousso and Hawking [2] ( in short, $\mathrm{BH}$ ) calculated the probability of the quantum creation of a universe with a pair of primordial black holes in $(3+1)$ dimensional universe. In the paper they considered two different euclidean space-time : (1) a universe with space-like sections with topology $S^{3}$ and (2) a universe with space-like section with topology $S^{1} \times S^{2}$, as is obtained in the Schwarzschild- de Sitter solution. The first kind of spatial structure describes an inflationary (de Sitter) universe without black hole while the second kind describes a Nariai universe [3], an inflationary universe with a pair of black holes. BH considered in their paper a massive scalar field which provided an effective cosmological constant for a while through a slow-rolling potential (mass-term). Chao [4] has studied the creation of a primordial black hole and Green and Malik [5] studied

the primordial black holes production during reheating. Paul et al. [6] following the approach of $\mathrm{BH}$ studied the probability of creation of $\mathrm{PBH}$ including $R^{2}$-term in the Einstein action and found that the probability is very much suppressed in the $R^{2}$-theory. It is well known that the theory with higher order Lagrangian 
are conformally equivallent to Einstein gravity with a matter sector containing a minimally coupled, self interacting scalar field. However, the renormalization of higher loop contributions introduces terms into the effective action that are higher than quadratic in $R$. Consequently it is important to study the effects of these terms to study quantum creation of a universe with a pair of $\mathrm{PBH}$. It is important to confine with terms upto cubic terms only as for a polynomial Lagrangian

$$
f(R)=\sum_{n=1}^{N} \lambda_{n} R^{n}
$$

the form of the potential is extremely complicated. It was shown by Henk van Elst et al. [7] that $N=2$ contribution is rather special in four dimensions, while $R^{3}$-term gives a more generic purturbation. The qualitative behavior of the potential $V(\phi)$ does not change relative to the $R^{3}$ term even when terms with $N>3$ are considered. We, therefore, consider term upto $R^{3}$ in the action and look for probability of a pair of $\mathrm{PBH}$ in the early universe. We calculate the probabilities for the creation of a universe with two types of topology namely, $R \times S^{3}$ topology and $R \times S^{1} \times S^{2}$ topology which accommodates a pair of primordial black holes. To calculate the probabilities for these spatial topologies, we use a semiclassical approximation for the evaluation of the euclidean path integrals. The condition that a classical spacetime should emerge, to a good approximation, at a large Lorentzian time was selected by a choice of the path of the time parameter $\tau$ along the $\tau^{R e}$ axis from 0 to $\frac{\pi}{2 H}$ and then continues along the $\tau^{i m}$ axis. The wave-function of the universe in the semiclassical approximation is given by

$$
\Psi_{o}\left[h_{i j}, \Phi_{\partial M}\right] \approx \sum_{n} A_{n} e^{-I_{n}}
$$

where the sum is over the saddle points of the path integral, and $I_{n}$ denotes the 
corresponding Euclidean action. The probability measure of the creation of PBH is

$$
P\left[h_{a b}, \Phi_{\partial M}\right] \sim e^{-2 I^{R e}}
$$

where $h_{a b}$ is the boundary metric and $I^{R e}$ is the real part of the action corresponding to the dominant saddle point, i.e. the classical solution satisfying the Hartle-Hawking (henceforth, HH) boundary conditions [8]. It was believed that all inflationary models lead to $\Omega_{o} \sim 1$ to a great accuracy. This view was modified after it was discovered that there is a special class of inflaton effective potentials which may lead to a nearly homogeneous open universe with $\Omega_{o} \leq 1$ at the present epoch. Cornish et al. $[9,10]$ studied the problem of pre-inflationary homogeneity and outlines the possibility of creation of a small, compact, negatively curved universe. We show that a universe with $S^{3}$-topology may give birth to an open inflation.

The paper is organised as follows : in sec. II we write the gravitational action for a higher derivative theories and obtain gravitational instanton solutions and in sec. III we use the action to estimate the relative probability of the two types of the universes and in sec. IV we give a brief discussion.

\section{GRAVITATIONAL INSTANTON SOLUTIONS WITH OR WITHOUT A PAIR OF PRIMORDIAL BLACK HOLES:}

We consider a Euclidean action containing higher derivative terms which is given by

$$
I_{E}=-\frac{1}{16 \pi} \int d^{4} x \sqrt{g} f(R)-\frac{1}{8 \pi} \int_{\partial M} d^{3} x \sqrt{h} K f^{\prime}(R)
$$


where $g$ is the 4-dimensional Euclidean metric, $f(R)=R+\alpha R^{2}+\beta R^{3}-2 \Lambda, R$ is the Ricci scalar and $\Lambda$ is the 4-dimensional cosmological constant. In the gravitational surface term, $h_{a b}$ is the boundary metric and $K=h^{a b} K_{a b}$ is the trace of the second fundamental form of the boundary $\partial M$ in the metric. The second term is the contribution from $\tau=0$ back in the action. It vanishes for a universe with $S^{3}$ topology, but gives a non vanishing contribution for $S^{1} \times S^{2}$-topology.

\section{(A) Topology $S^{3}$, the de Sitter spacetime :}

In this section we study vacuum solutions of the Euclidean Einstein equation with a cosmological constant in four dimensions. We now look for a solution with spacelike section $S^{3}$. Hence we choose the four dimensional metric ansatz

$$
d s^{2}=d \tau^{2}+a^{2}(\tau)\left[d x_{1}^{2}+\sin ^{2} x_{1} d \Omega_{2}^{2}\right]
$$

where $a(\tau)$ is the scale factor of a four dimensional universe and $d \Omega_{2}^{2}$ is a line element on the unit two sphere. The scalar curvature is given by

$$
R=-6\left[\frac{\ddot{a}}{a}+\left(\frac{\dot{a}^{2}}{a^{2}}-\frac{1}{a^{2}}\right)\right]
$$

where an overdot denotes differentiation with respect to $\tau$. We rewrite the action (2), including the constraint through a Lagrangian multiplier $\beta$ and obtain

$$
I_{E}=-\frac{\pi}{8} \int\left[f(R) a^{3}-\beta\left(R+6 \frac{\ddot{a}}{a}+6 \frac{\dot{a}^{2}-1}{a^{2}}\right)\right] d \tau-\frac{1}{8 \pi} \int_{\partial M} d^{3} x \sqrt{h} K f^{\prime}(R) .
$$

Varying the action w.r.t. $\mathrm{R}$, we determine

$$
\beta=a^{3} f^{\prime}(R)
$$

Substituting the above equation, treating $a$ and $R$ as independent variables we get

$$
I_{E}=-\frac{\pi}{8} \int_{\tau=0}^{\tau \frac{\pi}{2 H_{o}}}\left[a^{3} f(R)-f^{\prime}(R)\left(a^{3} R-6 a \dot{a}^{2}-6 a\right)+6 a^{2} \dot{a} \dot{R} f^{\prime \prime}(R)\right] d \tau
$$




$$
-\frac{3 \pi}{4}\left[\dot{a} a^{2} f^{\prime}(R)\right]_{\tau=0}
$$

here we have eliminated $\ddot{a}$ by integration by parts. The field equations can now be obtained by varying $I_{E}$ with respect to $a$ and $R$, giving

$$
\begin{gathered}
f^{\prime \prime}(R)\left[R+6 \frac{\ddot{a}}{a}+6 \frac{\dot{a}^{2}-1}{a^{2}}\right]=0, \\
2 f^{\prime \prime \prime}(R) \dot{R}^{2}+2 f^{\prime \prime}(R)\left[\ddot{R}+2 \frac{\dot{a}}{a} \dot{R}\right]+f^{\prime}(R)\left[4 \frac{\ddot{a}}{a}+2 \frac{\dot{a}^{2}}{a^{2}}-\frac{2}{a^{2}}+R\right]-f(R)=0 .
\end{gathered}
$$

We now consider $f(R)=R+\alpha R^{2}+\beta R^{3}-2 \Lambda$ and obtain an instanton solution which is given by

$$
a=\frac{1}{H_{o}} \sin H_{o} \tau
$$

where $H_{o}^{2}[\beta, \Lambda]$ is determined from the constraint equation $432 \beta H_{o}^{6}-3 H_{o}^{2}+\Lambda=0$. We note that this solution satisfies the $\mathrm{HH}$ no boundary conditions viz., $a(0)=0$, $\dot{a}(0)=1$. One can choose a path along the $\tau^{R e}$ axis to $\tau=\frac{\pi}{2 H}$, the solution describes half of the Euclidean de Sitter instanton $S^{3}$. Analytic continuation of the metric (3) to Lorentzian region, $x_{1} \rightarrow \frac{\pi}{2}+i \sigma$, gives

$$
d s^{2}=d \tau^{2}+a^{2}(\tau)\left[-d \sigma^{2}+\cosh ^{2} \sigma d \Omega_{2}^{2}\right]
$$

which is a spatially inhomogeneous de Sitter like metric. However, if one sets $\tau=i t$ and $\sigma=i \frac{\pi}{2}+\chi$, the metric becomes

$$
d s^{2}=-d t^{2}+b^{2}(t)\left[d \chi^{2}+\sinh ^{2} \chi d \Omega_{2}^{2}\right]
$$

where $b(t)=-i a(i t)$. It describes the creation of an open inflationary universe. The real part of the Euclidean action corresponding to the solution calculated by following the complex contour of $\tau$ suggested by $\mathrm{BH}$ is given by

$$
I_{S^{3}}^{R e}=-\frac{\pi}{2 H_{o}^{4}}\left[24 \alpha H_{o}^{4}+4 H_{o}^{2}-\Lambda\right]
$$


With the chosen path for $\tau$, the solution describes half the de Sitter Instanton with $S^{4}$ topology, joined to a real Lorentzian hyperboloid of topology $R^{1} \times S^{3}$. It can be joined to any boundary satisfying the condition $a_{\partial M}>0$. For $a_{\partial M}>H_{o}^{-1}$, the wave function oscillates and predicts a classical space-time. We note the following cases : $\bullet \beta=0$ one obtains $H_{o}^{2}=\Lambda / 3$ and $R=4 \Lambda$ which corresponds to the result obtained by Paul et al. [6]. • $\beta \neq 0$ and one gets now instanton solution even with a cosmological constant i.e., $\Lambda=0$ giving $H_{o}^{2}=\frac{1}{12 \sqrt{\beta}}$. This solution is interesting as with $N \leq 2$ in the polynomial $f(R)$ one always requires a cosmological constant to get such an instanton.

(B) Topology $S^{1} \times S^{2}$ :

In this section we consider Euclidean Einstein equation and look for a universe with $S^{1} \times S^{2}$-spacelike sections as this topology accommodates a pair of black holes. The corresponding ansatz for $(1+1+2)$ dimensions is given by

$$
d s^{2}=d \tau^{2}+a^{2}(\tau) d x^{2}+b^{2}(\tau) d \Omega_{2}^{2}
$$

where $a(\tau)$ is the scale factor of two sphere and $b(\tau)$ is the scale factor of the two sphere given by the metric

$$
d \Omega_{2}^{2}=d \theta^{2}+\sin ^{2} \theta d \phi^{2}
$$

The scalar curvature is given by

$$
R=-\left[2 \frac{\ddot{a}}{a}+4 \frac{\ddot{b}}{b}+2\left(\frac{\dot{b}^{2}}{b^{2}}-\frac{1}{b^{2}}\right)+4 \frac{\dot{a} \dot{b}}{a b}\right] .
$$

The Euclidean action (2) becomes

$$
I_{E}=-\frac{\pi}{2} \int\left[f(R) a b^{2}-\beta\left(R+2 \frac{\ddot{a}}{a}+4 \frac{\ddot{b}}{b}+4 \frac{\dot{a} \dot{b}}{a b}+2 \frac{\dot{b}^{2}}{b^{2}}-\frac{2}{b^{2}}\right)\right] d \tau
$$




$$
-\frac{1}{8 \pi} \int_{\partial M} \sqrt{h} d^{3} x K f^{\prime}(R) .
$$

One can determine $\beta$ as is done before and obtains

$$
\begin{gathered}
I_{S^{1} \times S^{2}}=-\frac{\pi}{2} \int_{\tau=0}^{\tau_{\partial M}}\left[f(R)-f^{\prime}(R)\left(R-4 \frac{\dot{a} \dot{b}}{a b}-2 \frac{\dot{b}^{2}}{b^{2}}-\frac{2}{b^{2}}\right)+2 f^{\prime \prime}(R) \dot{R}\left(\frac{\dot{a}}{a}+2 \frac{\dot{b}}{b}\right)\right] \\
a b^{2} d \tau-\pi\left[\left(\dot{a} b^{2}+2 a b \dot{b}\right) f^{\prime}(R)\right]_{\tau=0} .
\end{gathered}
$$

Variation of the action with respect to the scale factors $R, a$ and $b$ gives the following field equations

$$
\begin{aligned}
& f^{\prime \prime}(R)\left(R+2 \frac{\ddot{a}}{a}+4 \frac{\ddot{b}}{b}+4 \frac{\dot{a} \dot{b}}{a b}+2 \frac{\dot{b}^{2}}{b^{2}}-\frac{2}{b^{2}}\right), \\
& 2 f^{\prime \prime \prime}(R) \dot{R}^{2}+2 f^{\prime \prime}(R)\left(\ddot{R}+2 \dot{R} \frac{\dot{b}}{b}\right)+f^{\prime}(R)\left(R+4 \frac{\ddot{b}}{b}+2 \frac{\dot{b}^{2}-1}{b^{2}}\right)-f(R)=0, \\
& 2 f^{\prime \prime \prime}(R) \dot{R}^{2}+2 f^{\prime \prime}(R)\left(\dot{R}\left(\frac{\dot{a}}{a}+\frac{\dot{b}}{b}\right)+\ddot{R}\right)+f^{\prime}(R)\left(R+2 \frac{\ddot{a}}{a}+2 \frac{\ddot{b}}{b}+2 \frac{\dot{a} \dot{b}}{a b}\right) \\
& -f(R)=0 .
\end{aligned}
$$

With $f(R)=R+\alpha R^{2}+\beta R^{3}-2 \Lambda$ one obtains an instanton solution from the field equations (17)-(19) which is given by

$$
\begin{gathered}
a=\frac{1}{H_{o}} \sin \left(H_{o} \tau\right), \quad b=H_{o}^{-1}, \\
R=4 H_{o}^{2}
\end{gathered}
$$

where $H_{o}$ satisfies the equation

$$
16 \beta H_{o}^{6}-H_{o}^{2}+\Lambda=0 .
$$

We note the following : (i) when $\Lambda=0, H_{o}^{4}=\frac{1}{16 \beta}$ and $\alpha=-3 \sqrt{\beta}$ which demands a positive coupling constant $\beta$, (ii) when $\Lambda \neq 0$ then $\alpha= \pm 3 \sqrt{\beta}$ which demands a positive coupling constant $\beta$ but $\alpha$ may take either sign, (iii) when $\beta=0, \Lambda=H_{o}^{2}$ 
corresponds to the solution obtained by Paul et al. [6], and (iv) $\alpha=0$ and $\beta=0$ leads to $\Lambda=H_{o}^{2}$ which corresponds to BH solution [2]. These solutions satisfy the $\mathrm{HH}$ boundary conditions $a(0)=0, \dot{a}(0)=1, b(0)=b_{o}, \dot{b}(0)=0$. Analytic continuation of the metric (13) to Lorentzian region, i.e., $\tau \rightarrow i t$ and $x \rightarrow \frac{\pi}{2}+i \sigma$ yields

$$
d s^{2}=-d t^{2}+c^{2}(t) d \sigma^{2}+H_{o}^{-2} d \Omega_{2}^{2}
$$

where $c(t)=-i a(i t)$. In this case the analytic continuation of time and space do not give an open inflationary universe. The corresponding Lorentzian solution is given by

$$
\begin{gathered}
\left.a\left(\tau^{I m}\right)\right|_{\tau^{R e}=\frac{\pi}{2 H}}=H^{-1} \cosh H \tau^{I m}, \\
\left.b\left(\tau^{I m}\right)\right|_{\tau^{R e}=\frac{\pi}{2 H}}=H^{-1}
\end{gathered}
$$

Its space like sections can be visualised as three spheres of radius $a$ with a hole of radius $b$ punched through the north and south poles. The physical interpretation of the solution is that of two - spheres containing two black holes at opposite ends. The black holes have the radius $H^{-1}$ accelerates away from each other with the expansion of the universe. The real part of the action can now be determined following the contour suggested by $\mathrm{BH}[2]$, and it is given by

$$
I_{S^{1} X S^{2}}^{R e}=-\frac{\pi}{H_{o}^{4}}\left[8 \alpha H_{o}^{4}+4 H_{o}^{2}-3 \Lambda\right] .
$$

where $H_{o}^{2}$ satisfies the constraint

$$
16 \beta H_{o}^{6}-H_{o}^{2}+\Lambda=0
$$

The solution (20) describes a universe with two black holes at the poles of a two sphere. However, unlike the $\mathrm{BH}$ case we are getting vanishing contribution of the action in this case from the surface term. 


\section{EVALUATION FOR THE PROBABILITY FOR PBH :}

In the previous section we have calculated the actions for inflationary universe with or without a pair of black holes. We now compare the probability measure. The probability for creation of a higher dimensional de Sitter universe may be obtained from the action (6). Thus, the probability for nucleation of a higher dimensional universe without $\mathrm{PBH}$ is given by

$$
P_{S^{3}} \sim e^{\frac{\pi}{H_{o}^{4}}\left[24 \alpha H_{o}^{4}+4 H_{o}^{2}-\Lambda\right]}
$$

However for an inflationary universe with a pair of black holes the corresponding probability of nucleation can be obtained from the action (16). The corresponding probability is

$$
P_{S^{1} \times S^{2}} \sim e^{\frac{2 \pi}{H_{o}^{4}}\left[8 \alpha H_{o}^{4}+4 H_{o}^{2}-3 \Lambda\right]}
$$

We now describe the spacial cases of the general result:

- when $\alpha=0, \beta=0$ one recovers the result obtained by Bousso and Hawking

$$
P_{S^{3}} \sim e^{\frac{3 \pi}{\Lambda}}, \quad P_{S^{1} \times S^{2}} \sim e^{\frac{2 \pi}{\Lambda}}
$$

Thus with a positive cosmological constant the probability for a universe with PBH is less than that without $\mathrm{PBH}$.

- when $\alpha \neq 0, \beta=0$ one determines $H_{o}^{2}=\Lambda$ and the probabilities are

$$
P_{S^{3}} \sim e^{\frac{3 \pi}{\Lambda}+24 \pi \alpha}, \quad P_{S^{1} \times S^{2}} \sim e^{\frac{2 \pi}{\Lambda}+16 \pi \alpha} .
$$

In this case with a positive cosmological constant and a positive $\alpha>0$, de Sitter universe is more probable [6].

$$
P_{S^{3}} \sim e^{\frac{3 \pi}{\Lambda}}, \quad P_{S^{1} \times S^{2}} \sim e^{\frac{2 \pi}{\Lambda}}
$$


However negative values of $\alpha<-\frac{1}{8 \Lambda}$ could lead to an interesting possibilities as the probability for a universe with $\mathrm{PBH}$ in this case is more.However, the case $\alpha<0$ leads to a classical instability in $R^{2}$-theory.

- when $\alpha \neq 0, \beta \neq 0$ one determines obtains instanton solution even with $\Lambda=0$ and we get $H_{o}^{2}=\sqrt{\frac{1}{144 \beta}}$ which leads to the probabilities

$$
P_{S^{3}} \sim e^{8 \pi(3 \alpha+6 \sqrt{\beta})}, \quad P_{S^{1} \times S^{2}} \sim e^{16 \pi(\alpha+2 \sqrt{\beta})} .
$$

It is evident that one requires $\beta>0$ to obtain instanton but the values of $\alpha$ may be positive or negative. When $\alpha>-2 \sqrt{\beta}$ or $|\alpha|<2 \sqrt{\beta}$ one obtains that a universe without $\mathrm{PBH}$ is more probable. In this case interesting possibilities emerges when $\alpha<-2 \sqrt{\beta}$ or $|\alpha|>2 \sqrt{\beta}$.

\section{DISCUSSIONS :}

In this work we have evaluated the probability for primordial black holes pair creation in a higher derivative theories. In section II, we have obtained the gravitational instanton solutions in two cases : a universe with (i) $R \times S^{3}$ - topology and a universe with (ii) $R \times S^{1} \times S^{2}$-topology respectively. The Euclidean action is then evaluated. We found that the probability of a universe with $R \times S^{3}$ topology turns out to be much lower than a universe universe with topology $R \times S^{1} \times S^{2}$ in the $R^{2}$-theory unless $\alpha<-\frac{1}{8 \alpha}$. It may be mentioned here that one gets a regular instanton in $S^{4}$ - topology if there are no black holes. The existence of black holes restricts such a regular topology. The result obtained here on the probability of creation of a universe with a pair of primordial black holes is found to be strongly suppressed if one extends the theory with $R^{3}$-term in the action under the constraints (i) $\alpha>-2 \sqrt{\beta}$ or (ii) $|\alpha|<2 \sqrt{\beta}$ when $\Lambda=0$. Thus one interesting result is that in the framework 
of $R^{3}$-theory we get de Sitter instantons with $S^{3}$ and $S^{1} \times S^{2}$ topologies even without a cosmological constant. Thus one obtains $\mathrm{PBH}$ in the theory even without a cosmological constant in $R^{3}$ theory. It is interesting to note here that analytic continuation of a $R \times S^{3}$ metric considered here to Lorentzian region leads to an open 3 - space. One obtains Hawking-Turok $[11,12]$ type open inflationary universe in this case. In the other type of topology an open inflation is not permitted. A detail study of an open inflationary universe will be discussed elsewhere. Thus in a polynomial Lagrangian in $R$-theory, quantum creation of PBH seems to be suppressed in the minisuperspace cosmology for some values of the parameters in the action.

\section{Acknowledgement :}

The work is suported by Minor Research grant of the University Grants Commission, New Delhi and North Bengal University (NBU). BCP would like to thank the Inter-University University Centre for Astronomy and Astrophysics (IUCAA), Pune for providing facilities to carry out the work and AS would like to thank the Physics Department, North Bengal University and to S. Mukherjee for providing IRC facilities at NBU to carry out a part of the work. 


\section{References}

[1] S. W. Hawking, Comm. Math. Phys. 43, 199 (1975).

[2] R. Bousso and S. W. Hawking, Phys. Rev. D 52, 5659 (1995).

[3] H. Nariai,Sci. Rep. Tohoku Univ. 35, 62 (1951).

[4] W. Z. Chao, Int. J. Mod. Phys. D6, 199 (1997).

[5] A. M. Green and K. A. Malik, Phys. Rev. D 64, 021301 (2001).

[6] B. C. Paul, G. P. Singh, A. Beesham and S. Mukherjee, Mod. Phys. Letts. A 13, 2289 (1998).

[7] Henk van Elst, J. E. Lidsey and R. Tavakol, Class. Quantum Grav. 11, 2483 (1994).

[8] J. B. Hartle and S. W. Hawking,Phys. Rev. D 28, 2960 (1983).

bibitemkn : 9 N. J. Cornish, D. N. Spergel and G. D. Starkman, Phys. Rev. Letts. 77, 215 (1996) ; Class. Quantum Grav. 15, 2657 (1998).

[9] N. J. Cornish and D. N. Spergel, 'A small Universe after all? ' - astro ph/9906401.

[10] S. W. Hawking and N. G. Turok, Open Inflation without false Vacuum, hepth/9802030.

[11] N. G. Turok and S. W. Hawking, Open Inflation, the four form and the cosmological constant, hep-th/9803156. 